\title{
New DQ white dwarfs in the Sloan Digital Sky Survey DR4: confirmation of two sequences ${ }^{\star}$
}

\author{
D. Koester and S. Knist
}

\author{
Institut für Theoretische Physik und Astrophysik, University of Kiel, 24098 Kiel, Germany \\ e-mail: koester@astrophysik.uni-kiel.de
}

Received 27 March 2006 / Accepted 26 April 2006

ABSTRACT

\begin{abstract}
Context. DQ white dwarfs show atomic or molecular carbon features in their spectra. The atmosphere consists of helium and the carbon is believed to be dredged-up to the surface by the deepening helium convection zone.

Aims. We want to identify new DQ in the Data Release 4 of the Sloan Digital Sky Survey, determine the effective temperatures and carbon abundances and search for systematic trends between these parameters as indication of the origin of this class.

Methods. Photometric selection criteria are developed and applied to the catalog to identify candidates, which are in a final step confirmed by visual inspection of spectra. The parameters are determined through comparison with theoretical spectra and colors. Results. Our final DQ catalog contains 65 stars. 40 of these are new identifications, the other 25 have been described as DQ before in the literature. We derive effective temperatures and carbon abundances for 60 of these stars. The majority of stars defines a clear sequence in the $\mathrm{C}$ abundance vs. $T_{\text {eff }}$ diagram, with high abundances found at high $T_{\text {eff }}$ and vice versa. We also confirm with high significance a second sequence with an abundance about 1 dex higher at the same $T_{\text {eff }}$, and discuss the nature and possible origin of the high-C sequence.
\end{abstract}

Key words. stars: white dwarfs - stars: abundances

\section{Introduction}

White dwarfs of spectral type DQ are defined as showing atomic or molecular features of carbon in a helium atmosphere. Contrary to other metals (notably $\mathrm{Ca}$ ) found in cool white dwarfs, which are thought to be provided through an accretion process from outside the star, the most widely accepted explanation for the DQs is dredge-up from the underlying carbon/oxygen core through the expanding He convection zone (Koester et al. 1982; Pelletier et al. 1986). A detailed summary of the basic properties of this class and earlier references to spectroscopic analyses has been given recently in a comprehensive paper by Dufour et al. (2005, =DB05 henceforth) and need not be repeated here.

In addition to the general understanding of the nature, evolutionary relations and origin of the peculiar spectral types of white dwarfs, the DQs are of special interest, because they provide information about the deeper layers of the stars. The transition between the outer helium layer and the carbon/oxygen core - the result of the helium burning in the progenitor - is not abrupt, but rather gradual as determined by the diffusion equilibrium (Koester et al. 1982). We can predict the structure of this transition quantitatively (Pelletier et al. 1986), as well as the depth of the helium convection zone at a given effective temperature and mass of the star. The observed carbon abundance in the atmosphere is then directly related to the thickness of the helium layer remaining from the previous nuclear evolution, which is a very important parameter for the understanding of stellar evolution. The first estimate of this thickness by Pelletier et al. $(1986)-\log M_{\mathrm{He}} / M \approx-3.75$ - was much thinner than predicted by evolutionary calculations of the AGB phase. However,

$\star$ Appendix $\mathrm{A}$ is only available in electronic form at http://www . edpsciences.org the recent abundance determinations by DB05, together with the new models of the outer layers by Fontaine \& Brassard (2005) are in much better agreement with theoretical expectations. Nevertheless, there are still many problems understanding the detailed connection of the DQ stars to possible helium-rich progenitors (Althaus et al. 2005; Scóccola et al. 2006).

When searching for correlations of DQ parameters, e.g. between atmospheric carbon abundance and effective temperature, it is very helpful to have available a homogeneous sample of observations. It has been found in previous studies that abundances determined from atomic lines in the UV or molecular bands in the optical may sometimes differ significantly (e.g. Provencal et al. 2002), possibly blurring any correlation if only one or the other observation is available for different objects (Weidemann \& Koester 1995). Such a homogeneous sample is currently provided by the Sloan Digital Sky Survey, and DB05 provides the first quantitative analysis of 40 DQs from the Data Release 1 (DR1). In this paper we report similar results for 40 new DQs (and 20 previously known) extracted from DR4 (Adelman-McCarthy et al. 2006).

\section{Selection of $D Q$ candidates}

DR4 of the SDSS contains spectra for approximately 240000 objects classified as "point source" or "unknown". In order to narrow down this huge database to tractable numbers we have applied three consecutive selection steps, the first two based on the SDSS photometry, the last on the spectra.

\subsection{DQ regions in SDSS color space and theoretical models}

Theoretical SDSS colors can be calculated from synthetic spectra by convolving them with the 5 ugriz SDSS band passes. Our 


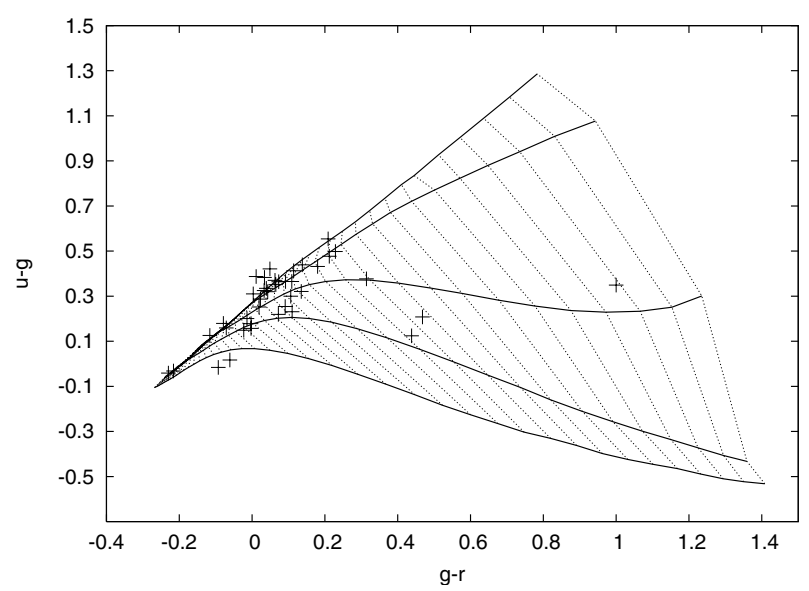

Fig. 1. Theoretical SDSS color grid for DQ white dwarfs. Thick continuous lines are lines of constant carbon abundance from $[\mathrm{C} / \mathrm{He}]=-8.0$ (top) to -4 (bottom), step 1.0. Thin dashed: lines of constant $T_{\text {eff }}$ from $4400 \mathrm{~K}$ (right) to $13000 \mathrm{~K}$ (left) in steps of $200 \mathrm{~K}$. Crosses show the observed DQ stars from Harris et al. (2003) and Kleinman et al. (2004).

model grid is similar to that described in Carollo et al. (2003), but extended to cover effective temperatures from $13000 \mathrm{~K}$ to $4400 \mathrm{~K}$. Logarithmic $\mathrm{C}$ abundance by number relative to $\mathrm{He}$ $(=[\mathrm{C} / \mathrm{He}])$ range from -8 to -4 in steps of 0.5 . The standard grid used here has a surface gravity of $\log g=8.00$, but additional grids were calculated with 7.5 and 8.5 for comparison. The models are fully blanketed, fully including the line and molecular band absorption in the calculation of the atmospheric structure. Non-ideal effects in the form of a lowering of the dissociation energy of the $\mathrm{C}_{2}$ molecule is included in a very approximate way.

The transmission curves for the five filters (for airmass 1.3 as recommended) were obtained from the SDSS web sites; the zero-points for the magnitudes were set to correspond to the AB system of magnitudes (Fukugita et al. 1996) except for small changes to $u, i, z(-0.04,0.01,0.02)$ similar to those discussed in Eisenstein et al. (2006).

The dependence on $T_{\text {eff }}$ and $[\mathrm{C} / \mathrm{He}]$ is strongest in the $u-$ $g$ vs. $g-r$ two-color diagram and much less in the remaining independent indices used, $r-i$ and $i-z$. Figure 1 shows this diagram with the theoretical grid, and 40 observed DQs from Harris et al. (2003) and Kleinman et al. (2004), which indeed fall in or very near the theoretical grid. Note that we have not applied any correction for galactic extinction (see DB05).

Using this information about the position of DQs in the SDSS color space, we have, as a first selection step, extracted from the SDSS database all objects in the "point source" and "unknown" categories falling into the region defined by

$$
-0.8<u-g<1.5 \quad \text { and } \quad-0.8<g-r<1.5 \text {, }
$$

resulting in 86856 selected objects. This sample still contains large numbers of DA white dwarfs and quasars. We therefore have, in a second step, refined the color region using a polygon in the $u-g, g-r$ plane. The polygon area follows rather closely the outline of the theoretical grid, except at the hot end. Since the grid converges towards a single line (the models are much less dependent on the $\mathrm{C}$ abundance) the polygon at the hot end is significantly wider in $u-g$. Applying this selection step narrowed the candidate sample down to 44928 objects.

\subsection{Selection in 4-dimensional color space}

Since we consider for the moment only the dependence of colors on $T_{\text {eff }}$ and $[\mathrm{C} / \mathrm{He}]$, it is obvious that the theoretical grid must define a 2-dimensional surface in the 4-dimensional color space $(u-g, g-r, r-i, i-z)$. That the variation is strongest in the first two components suggests that this surface might be approximated by a plane and this is confirmed by looking at different projections of the grid in two- or three-dimensional subspaces. We have therefore fitted a plane to the nodes of this grid using a $\chi^{2}$ minimization technique. The fit is extremely good, with typical deviations of grid points from the plane of $0.03 \mathrm{mag}$.

The equation of the plane is

$$
\begin{gathered}
\left(\begin{array}{l}
u-g \\
g-r \\
r-i \\
i-z
\end{array}\right)_{\text {plane }}=\left(\begin{array}{r}
0.00003 \\
-0.00001 \\
-0.09854 \\
-0.18105
\end{array}\right)+(g-r)\left(\begin{array}{r}
-0.00007 \\
1.00004 \\
0.53380 \\
0.19325
\end{array}\right) \\
+(u-g)\left(\begin{array}{r}
1.00000 \\
-0.00006 \\
-0.02747 \\
0.14871
\end{array}\right)
\end{gathered}
$$

where we have used the first two colors directly as the two variables for the plane. The reasonable region for DQs is given by the limits

$-0.55<u-g<1.3 \quad$ and $\quad-0.3<g-r<1.4$.

This plane representation of theoretical DQ colors was used in the final photometric selection step. We defined a minimum "distance" $d$ from the plane for each observed object by measuring each coordinate in units of their individual measurement errors

$d^{2}=\sum_{i=1}^{4}\left(\frac{c_{\mathrm{ob}}(i)-c_{\mathrm{th}}(i)}{\sigma(i)}\right)^{2}$

Here $c_{\mathrm{ob}}$ and $c_{\mathrm{th}}$ are the four observed and theoretical SDSS colors, and $\sigma$ the measurement error of the observation. We eliminated the majority of objects by using a limit of $d=6.5$. This limit had been determined from a test using the 40 DQs of Fig. 1. The final sample contained 6952 DQ candidates.

\subsection{Color space for different surface gravities}

We have also fitted the theoretical grids for $\log g=7.5$ and 8.5 in the same way. The resulting planes were practically indistinguishable from the $\log g=8.0$ plane, however, the lines of constant temperature or $\mathrm{C}$ abundance are slightly shifted. This corresponds to the well known fact for DQs that the parameters $\log g$ and $[\mathrm{C} / \mathrm{H}]$ are degenerate. They cannot both be determined from photometry (nor spectra), because a change of $\log g$ can always be very nearly compensated with a corresponding change of $[\mathrm{C} / \mathrm{He}]$, and one of the two has to be assumed to be able to proceed. Using the fact that most white dwarfs cluster around $0.6 M_{\odot}$, we have assumed $\log g=8$, as did DB05.

\subsection{Visual classification of the 6952 candidates}

Spectra for all remaining candidates were extracted from the SDSS database and inspected visually. More than 95\% turned out to be quasars, easily recognized by their strong and broad emission lines. Of the remaining objects some were DA white dwarfs (broad Balmer absorption), some galaxies with narrow 
Table 1. 40 new DQ white dwarfs. The first columns give the SDSS name and internal designation. Next is effective temperature determined from a fit to the photometry. The carbon abundance $[\mathrm{C} / \mathrm{He}]$ is determined from a model fit to the spectrum, keeping the temperature fixed. The final column gives a classification and remarks. WD 1105+412 and WD 0913+103 are reclassified from a DC classification in McCook \& Sion (1999). GD 311 is classified DC in Kawka \& Vennes (2006), but we believe very weak Swan bands to be present. SDSS J133127.04+670419.5 is classified DC in Kleinman et al. (2004).

\begin{tabular}{|c|c|c|c|c|c|c|c|}
\hline SDSS name & MJD & Plate & Fid & $T_{\text {eff }}[\mathrm{K}]$ & {$[\mathrm{C} / \mathrm{He}]$} & $\sigma[\mathrm{C} / \mathrm{He}]$ & Spectral type/Ref \\
\hline SDSS J074204.79+434835.7 & 53052 & 1736 & 139 & 7738 & -5.47 & 0.04 & DQ \\
\hline SDSS J084131.55+332915.6 & 52642 & 933 & 16 & 6810 & -6.62 & 0.05 & DQ \\
\hline SDSS J085239.66+042804.5 & 52670 & 1190 & 177 & 9555 & & & DQ \\
\hline SDSS J085506.62+063904.7 & 52668 & 1189 & 535 & 7337 & -5.99 & 0.01 & DQ \\
\hline SDSS J085709.01+060357.4 & 52668 & 1189 & 27 & 8210 & -5.00 & 0.02 & DQ \\
\hline SDSS J090449.73+395416.4 & 52703 & 1199 & 595 & 7319 & -5.74 & 0.05 & DQ \\
\hline SDSS J090514.78+090426.5 & 52973 & 1300 & 420 & 8861 & -4.89 & 0.01 & DQ $(\mathrm{H} \alpha ?)$ \\
\hline SDSS J090632.17+470235.8 & 52606 & 898 & 565 & 5070 & -4.31 & 0.01 & DQ (weak bands) \\
\hline SDSS J091602.73+101110.5 & 53050 & 1739 & 592 & 8715 & -4.81 & 0.01 & DQ \\
\hline SDSS J091830.27+484323.0 & 52637 & 900 & 429 & 8884 & -3.72 & 0.06 & DQ \\
\hline SDSS J092153.46+342136.9 & 52995 & 1274 & 216 & 8202 & -5.46 & 0.09 & DQ \\
\hline SDSS J092613.46+472521.1 & 52637 & 900 & 41 & 7261 & -6.41 & 0.06 & DQ \\
\hline SDSS J092909.03+331011.7 & 52991 & 1593 & 94 & 6361 & -5.57 & 0.01 & DQ \\
\hline SDSS J094014.65+090641.8 & 52993 & 1304 & 45 & 6169 & -7.34 & 0.05 & DQpec (rounded bands) \\
\hline SDSS J094115.18+090154.4 & 52993 & 1304 & 9 & 9122 & -4.73 & 0.02 & DQ \\
\hline SDSS J094138.08+441458.2 & 52672 & 1202 & 58 & 8113 & -5.42 & 0.06 & DQ \\
\hline SDSS J095934.95+453725.4 & 52703 & 942 & 446 & 7211 & -5.52 & 0.06 & DQ \\
\hline SDSS J100059.82+100531.7 & 53053 & 1308 & 307 & 7958 & -4.83 & 0.02 & DQ, WD $1105+412$ \\
\hline SDSS J101750.38+373637.5 & 52996 & 1427 & 216 & 7497 & -5.79 & 0.03 & DQ \\
\hline SDSS J101800.00+083820.3 & 52762 & 1237 & 621 & 7784 & -5.85 & 0.01 & DQ \\
\hline SDSS J102635.81+580714.8 & 52316 & 559 & 6 & 8879 & -4.56 & 0.03 & DQ \\
\hline SDSS J110759.46+405910.9 & 53046 & 1437 & 512 & 7169 & -6.45 & 0.02 & DQ \\
\hline SDSS J110912.21+424956.0 & 53053 & 1363 & 37 & 9402 & -4.84 & 0.08 & DQ, WD 0913+103 \\
\hline SDSS J112604.29+441938.6 & 53062 & 1365 & 564 & 7097 & -6.38 & 0.05 & DQ \\
\hline SDSS J113534.61+572451.7 & 53033 & 1310 & 485 & 7385 & -6.42 & 0.02 & GD 311, Kawka \& Vennes (2006) \\
\hline SDSS J115149.92+452729.8 & 53084 & 1368 & 503 & 8829 & -4.75 & 0.04 & DQ \\
\hline SDSS J122545.87+470613.0 & 53117 & 1451 & 35 & 6109 & -5.82 & 0.01 & DQ \\
\hline SDSS J123347.60+125346.1 & 53169 & 1616 & 429 & 7152 & -6.36 & 0.02 & DQ \\
\hline SDSS J130945.62+444541.0 & 53084 & 1375 & 391 & 8085 & -4.01 & 0.03 & DQ \\
\hline SDSS J131534.72+471108.9 & 53062 & 1461 & 428 & 7524 & -5.99 & 0.01 & DQ \\
\hline SDSS J131930.66+140137.1 & 53112 & 1773 & 105 & 7626 & -5.65 & 0.05 & DQ \\
\hline SDSS J133127.04+670419.5 & 51988 & 496 & 583 & 8899 & -4.91 & 0.06 & DQ (weak bands), Kleinman et al. (2004) \\
\hline SDSS J143144.83+375011.9 & 53089 & 1381 & 599 & 6173 & -6.97 & 0.02 & DQ \\
\hline SDSS J152812.05+513445.2 & 52378 & 795 & 319 & 7531 & -5.69 & 0.03 & DQ \\
\hline SDSS J153447.54+414559.4 & 53149 & 1679 & 616 & 7804 & -5.92 & 0.02 & DQZ (CaII) \\
\hline SDSS J161653.36+392444.4 & 52759 & 1336 & 572 & 7319 & -5.92 & 0.02 & DQ \\
\hline SDSS J165436.86+315754.4 & 52791 & 1176 & 238 & 7258 & -5.89 & 0.01 & DQ \\
\hline SDSS J171341.76+324009.1 & 52413 & 976 & 623 & 7901 & -5.36 & 0.01 & DQ \\
\hline SDSS J211130.04-003628.8 & 52431 & 985 & 35 & 7168 & -6.27 & 0.05 & DQ \\
\hline SDSS J213503.32+000318.4 & 52468 & 989 & 198 & 6413 & -6.78 & 0.03 & DQ \\
\hline
\end{tabular}

absorption lines. The DQs, the topic of this study, were identified through their molecular Swan bands. In total we found 65 DQs, of which 25 were already known in the literature, 36 are new detections, and 4 reclassifications from a spectral type of DC.

Tables 1 and 2 list all 65 DQ with their SDSS names, internal identifiers, and atmospheric parameters, determined as described in the next section. Most of the objects show only carbon features. SDSS J090514.78+090426.5 shows a feature near the position of $\mathrm{H} \alpha$, but nothing near the other Balmer lines, and the presence of hydrogen is highly uncertain. SDSS J153447.54+414559.4 shows Ca II H and K lines with equivalent widths of 2.1 and $1.0 \AA$. The wings are broad and the distance of the star is very likely less than $100 \mathrm{pc}$, implying a photospheric origin of the lines, probably due to accretion. SDSS J094014.65+090641.8 shows rounded bands similar to SDSS J223224.0-074434.3 in Harris et al. (2003).

\section{Atmospheric analysis of the DQ white dwarfs}

\subsection{Photometry}

The parameters $T_{\text {eff }}$ and $[\mathrm{C} / \mathrm{He}]$ were determined by minimizing in the sense of a $\chi^{2}$ the distance between the observed point in 4-dimensional color space with model colors interpolated on the $T_{\text {eff }}-[\mathrm{C} / \mathrm{He}]$ grid. $\chi^{2}$ minimization was obtained with the AMOEBA routine from Press et al. (1992). Since our sample contains 20 objects in common with DB05, we compare the derived $T_{\text {eff }}$ in Fig. 2 . The agreement in $T_{\text {eff }}$ with those from DB05, which are based on photometry and spectra is excellent, confirming that photometry alone already gives a reliable $T_{\text {eff }}$. From the differences between the two completely independent determinations we estimate a typical error for $T_{\text {eff }}$ of $190 \mathrm{~K}$. This is a much more realistic error than the internal errors from the $\chi^{2}$ routine; it is also very similar to the $T_{\text {eff }}$ error of $170 \mathrm{~K}$ cited 
Table 2. 25 rediscovered DQ white dwarfs in the same format as Table 1. 20 of these are analyzed also in DB05. Information on the remaining 5 can be found in the McCook \& Sion (1999) catalog (WD 1426+613), Liebert et al. (2003), Carollo et al. (2006), and Harris et al. (2003).

\begin{tabular}{lrrrrcrc}
\hline \hline SDSS name & MJD & Plate & Fid & $T_{\text {eff }}[\mathrm{K}]$ & {$[\mathrm{C} / \mathrm{He}]$} & $\sigma[\mathrm{C} / \mathrm{He}]$ & Spectral type/Ref \\
\hline SDSS J000011.57-085008.4 & 52143 & 650 & 450 & 8042 & -5.46 & 0.08 & \\
SDSS J000807.54-103405.6 & 52141 & 651 & 199 & 7768 & -5.66 & 0.06 & \\
SDSS J002531.50-110800.9 & 52145 & 653 & 86 & 8367 & -4.96 & 0.01 & \\
SDSS J015433.57-004047.2 & 51871 & 403 & 268 & 7435 & -5.89 & 0.03 & \\
SDSS J015441.75+140308.0 & 51877 & 430 & 558 & 6511 & -6.89 & 0.01 & \\
SDSS J032054.11-071625.4 & 51924 & 460 & 236 & 6266 & -5.45 & & \\
SDSS J033218.22-003722.1 & 51810 & 415 & 240 & 8600 & -4.62 & 0.03 & Liebert et al. (2003) \\
SDSS J090157.92+575135.9 & 51924 & 483 & 600 & & & & \\
SDSS J091922.18+023605.0 & 51929 & 473 & 458 & 11566 & & & Harris et al. (2003) \\
SDSS J093537.00+002422.0 & 52314 & 476 & 461 & 4958 & -6.19 & 0.02 & \\
SDSS J094004.64+021022.6 & 52026 & 477 & 493 & 7283 & -5.95 & 0.01 & \\
SDSS J095137.60+624348.7 & 51943 & 487 & 227 & 8388 & -5.11 & 0.10 & \\
SDSS J113359.94+633113.2 & 52059 & 597 & 139 & 12082 & & & \\
SDSS J114851.68-012612.8 & 52056 & 329 & 578 & 9174 & -3.73 & 0.01 & \\
SDSS J125359.61+013925.6 & 52026 & 523 & 252 & 8282 & -4.98 & 0.02 & Carollo et al. (2006) \\
SDSS J123752.12+415625.8 & 53090 & 1454 & 146 & 5846 & -5.51 & 0.01 & WD 1426+613 \\
SDSS J132858.20+590851.0 & 52411 & 959 & 504 & & & & \\
SDSS J142728.30+611026.4 & 52368 & 607 & 379 & 6427 & -6.83 & 0.01 & \\
SDSS J144407.25+043446.8 & 52026 & 587 & 418 & 9449 & -3.65 & 0.05 & \\
SDSS J144808.07-004755.9 & 51662 & 308 & 145 & 7063 & -6.50 & 0.04 & \\
SDSS J154810.66+562647.7 & 52072 & 617 & 551 & 8119 & -5.46 & 0.07 & \\
SDSS J155413.53+033634.5 & 52023 & 595 & 373 & 6512 & -6.94 & 0.03 & \\
SDSS J164328.54+400204.3 & 52050 & 630 & 386 & 7144 & -6.20 & 0.07 & \\
SDSS J165538.51+372247.1 & 52071 & 632 & 92 & 8997 & -4.75 & 0.06 & \\
SDSS J205316.34-070204.3 & 52176 & 636 & 267 & 6382 & -5.45 & 0.02 & \\
\hline
\end{tabular}

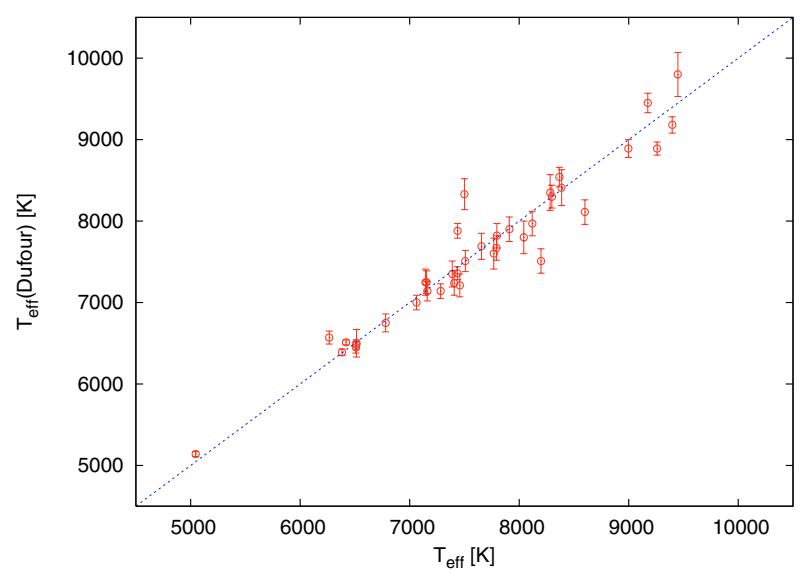

Fig. 2. Comparison of effective temperatures determined from the photometry with results from DB05.

by DB05, and we take this as our error estimate for $T_{\text {eff. }}$ On the other hand, the derived carbon abundances differ markedly, with a much higher scatter and also systematic differences. This is not really surprising, since the colors for different abundances converge at the high temperature end and a $[\mathrm{C} / \mathrm{He}]$ determination based on colors alone will be inaccurate to impossible. In addition, small uncertainties of the magnitude zero-points could shift the whole grid by a few hundreds of a magnitude, resulting in systematic changes. These abundances are not used further and therefore not shown here.

\subsection{Spectroscopy}

Fortunately, SDSS provides photometry and spectroscopy. The spectra were fitted with the theoretical spectra from our standard grid using the same methods used by us for other types of white dwarfs (Koester et al. 2001; Homeier et al. 1998). In principle the spectroscopic fit provides $T_{\text {eff }}$ and $[\mathrm{C} / \mathrm{He}](\log g=8$ is always assumed). However, since the flux calibration of the spectra is not completely reliable and the molecular features depend strongly on both, temperature and carbon abundance, the photometric observations provide a much more stringent constraint for the temperature. Our current codes cannot simultaneously fit spectra and photometric data. We have therefore chosen to keep the temperature fixed at the photometric solution (shown above to be reliable) and to determine only the carbon abundance from the spectra. Since the photometric temperature determination errors are smaller than those obtained by fitting both temperature and abundance from the spectra, this results also in smaller errors of the final $\mathrm{C}$ abundances. These abundances are given in Tables 1 and 2. For the five objects with missing entries in the $[\mathrm{C} / \mathrm{He}]$ column we could not obtain reasonable fits within our grid. Two examples for the spectral fits for the region around the strongest Swan bands are shown in Fig. 3. All fits of the 60 objects are shown in the electronic online version of the paper; the abundances are also given in Tables 1 and 2.

Figure 4 gives a comparison of our final results for the carbon abundance with DB05 for the common objects. The general agreement is very good, with a small systematic shift of about 0.2 dex, increasing from lower to high abundances. The $[\mathrm{C} / \mathrm{He}]$ abundances of DB05 are on average slightly higher than our values.

\section{Results and discussion}

We have identified 40 new DQ white dwarfs and rediscovered another 25 from the Data Release 4 of the Sloan Digital Sky Survey and presented effective temperatures and carbon abundances for 60 of them. This increases very significantly the 

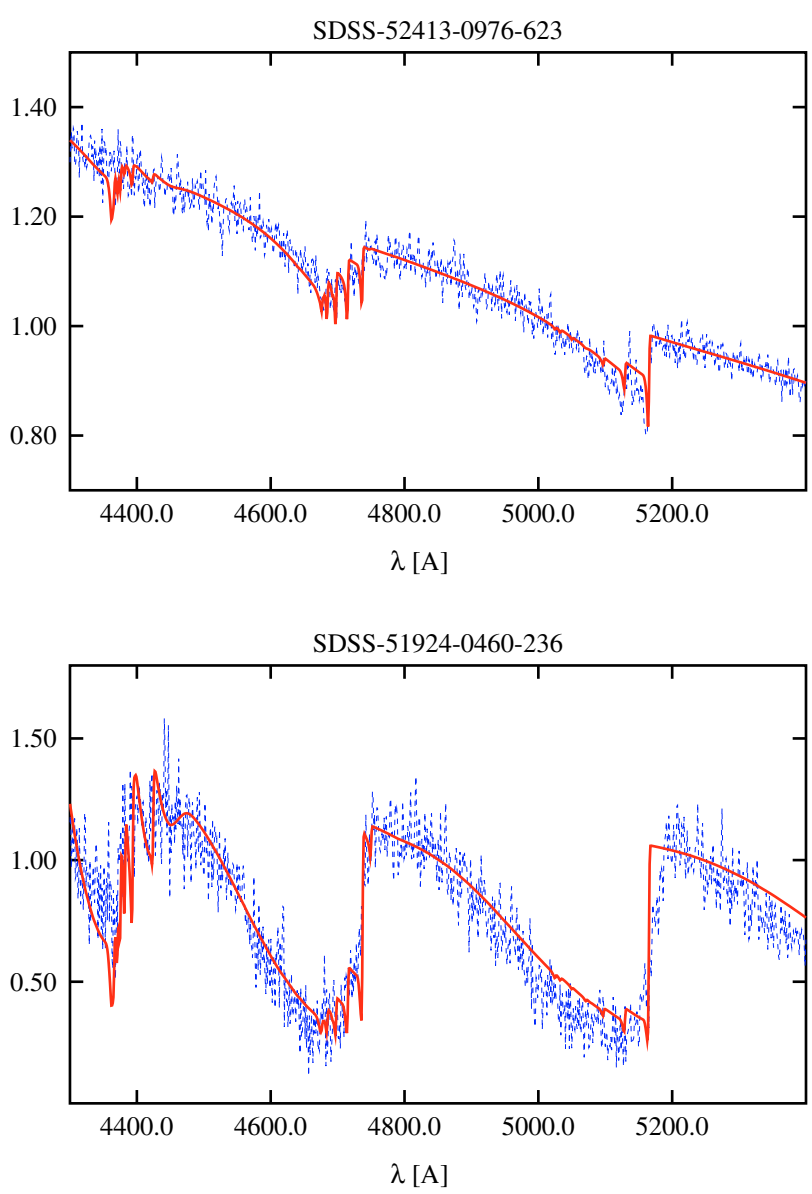

Fig. 3. Two examples of the spectral fitting for objects with molecular features of different strengths. Top: SDSS J171341.76+324009.1, bottom: SDSS J032054.11-071625.4. Note that the name used above the panels is the internal SDSS-MJD-PLATE-FID designation of the SDSS project, which can be cross-identified using Tables 1 and 2 . Vertical axis is relative intensity on an arbitrary scale.

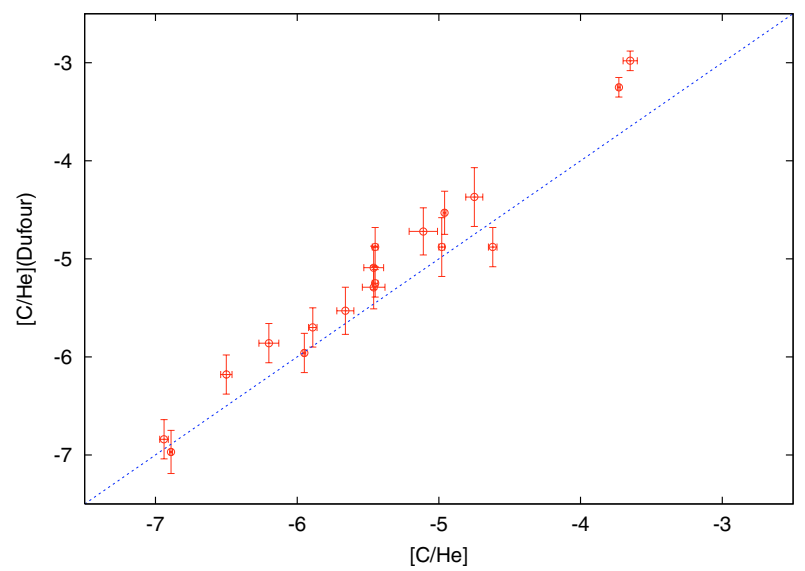

Fig. 4. Comparison of carbon abundances from spectral fitting with results from DB05.

number of DQs with a careful analysis using up-to-date model atmospheres. Particularly important is the fact that our sample is based on a completely homogeneous set of photometry and spectra. This is similar to the work of DB05, who however included also observations from other sources together with 40 SDSS objects in their sample. For the objects in common with their

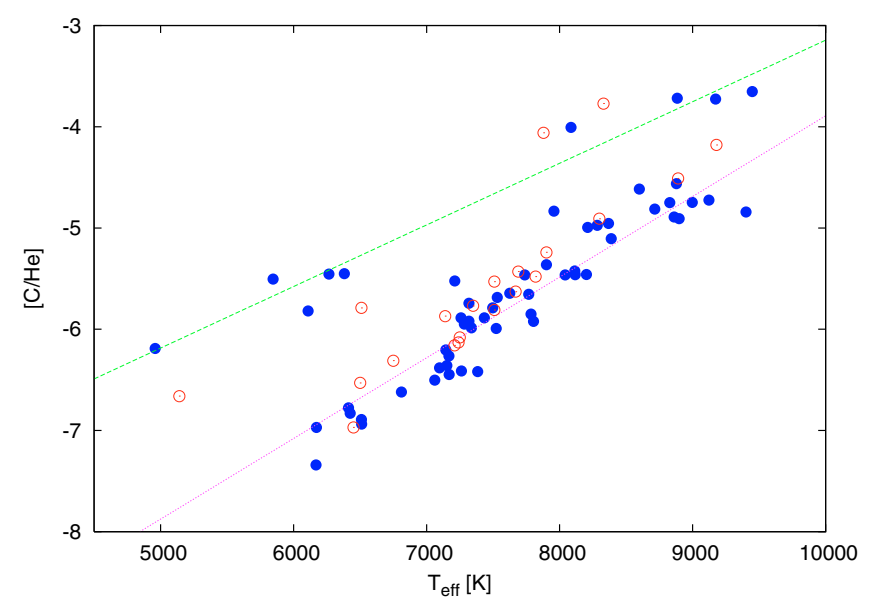

Fig. 5. Carbon abundance versus effective temperatures. Full circles are the results from this paper. We have added as open circles the results from DB05 for those objects not in our sample.

analysis we find good agreement for the parameters, with a slight offset in the abundances of the order of 0.2 dex.

The major result of this paper is shown in Fig. 5, which displays the carbon abundance versus effective temperature. In addition to our own results (shown as full circles) we have here included data from DB05 for those objects not included in our sample (open circles). We clearly confirm the three main conclusions of DB05:

- The paucity of objects with $T_{\text {eff }}$ below $6000 \mathrm{~K}$. Our sample adds only one object clearly below the apparent cutoff, also carbon-rich as the single cool objects in DB05.

- The clear sequence for the majority of the objects, from low $T_{\text {eff }}$, low $[\mathrm{C} / \mathrm{He}]$ to high $T_{\text {eff }}$, high $[\mathrm{C} / \mathrm{He}]$, indicated by the lower of the two regression line fits (these fits use only the results from this paper). The existence of this sequence was first pointed out by DB05 and shows the large advantage of using a homogeneous sample of good quality observations compared to e.g. the study of Weidemann \& Koester (1995). The extrapolation of the sequence to $10000 \mathrm{~K}$ predicts $[\mathrm{C} / \mathrm{He}]=-4.0$, in agreement with the theoretical calculations of Fontaine \& Brassard (2005) for a He-layer thickness of $10^{-4}$ of the stellar mass.

- The existence of a separate population of carbon-rich DQs, with an abundance about 1 dex higher than the primary sequence. This population seems to form a second sequence, which we have tentatively described with the upper regression line.

Based on one single object with a parallax and thus mass determination of $1.05 M_{\odot}$ in the upper sequence (G47-18 = WD 0856+33), DB05 tentatively suggested that all objects of this group are massive white dwarfs and might be the descendants of the hot DQ stars, which also seem to be massive, although that assertion is also only based on two objects (Liebert et al. 2003; Macdonald et al. 1998; Weidemann 2005). Stellar evolution calculations predict smaller He envelope masses for more massive progenitors (Kawai et al. 1988) and might lead to the dredge-up occurring at higher effective temperatures (Thejll et al. 1990). This would also be a natural explanation for the absence of the high-mass tail in the DB mass distribution (Beauchamp et al. 1996; Liebert et al. 2003).

While these conclusions seem rather speculative at present, it is obvious that further study of the large number of DQs with 
excellent observations coming from SDSS and other large scale surveys will provide important clues for the remaining open question of the origin and evolution of white dwarf surface compositions and spectral types. Of particular importance are parallax determinations for DQs on the high abundance sequence.

Acknowledgements. This study was partially supported by a grant from the Deutsche Forschungsgemeinschaft (KO731/21-1,-2), and would have been impossible without the SDSS. Funding for the Sloan Digital Sky Survey (SDSS) has been provided by the Alfred P. Sloan Foundation, the Participating Institutions, the National Aeronautics and Space Administration, the National Science Foundation, the US Department of Energy, the Japanese Monbukagakusho, and the Max Planck Society. The SDSS Web site is http://www.sdss.org/. The SDSS is managed by the Astrophysical Research Consortium (ARC) for the Participating Institutions. The Participating Institutions are The University of Chicago, Fermilab, the Institute for Advanced Study, the Japan Participation Group, The Johns Hopkins University, the Korean Scientist Group, Los Alamos National Laboratory, the Max-Planck-Institute for Astronomy (MPIA), the Max-Planck-Institute for Astrophysics (MPA), New Mexico State University, University of Pittsburgh, University of Portsmouth, Princeton University, the United States Naval Observatory, and the University of Washington. This research has made use of the SIMBAD database, operated at CDS, Strasbourg, France

\section{References}

Adelman-McCarthy, J. K., Agüeros, M. A., Allam, S. S., et al. 2006, ApJS, 162, 38

Althaus, L. G., Serenelli, A. M., Panei, J. A., et al. 2005, A\&A, 435, 631

Beauchamp, A., Wesemael, F., Bergeron, P., Liebert, J., \& Saffer, R. A. 1996, in Hydrogen deficient stars, ed. C. S. Jeffery, \& U. Heber, ASP Conf. Ser., 96, 295
Carollo, D., Koester, D., Spagna, A., Lattanzi, M. G., \& Hodgkin, S. T. 2003, A\&A, 400, L13

Carollo, D., Bucciarelli, B., Hodgkin, S. T., et al. 2006, A\&A, 448, 579

Dufour, P., Bergeron, P., \& Fontaine, G. 2005, ApJ, 627, 404

Eisenstein, D. J., Liebert, J., Koester, D., et al. 2006, ApJ, submitted

Fontaine, G., \& Brassard, P. 2005, in 14th European Workshop on White Dwarfs, ed. D. Koester, \& S. Moehler, ASP Conf. Ser., 334, 49

Fukugita, M., Ichikawa, T., Gunn, J. E., et al. 1996, AJ, 111, 1748

Harris, H. C., Liebert, J., Kleinman, S. J., et al. 2003, AJ, 126, 1023

Homeier, D., Koester, D., Hagen, H. J., et al. 1998, A\&A, 338, 563

Kawai, Y., Saio, H., \& Nomoto, K. 1988, ApJ, 328, 207

Kawka, A., \& Vennes, S. 2006 [arXiv: astro-ph/0601477]

Kleinman, S. J., Harris, H. C., Eisenstein, D. J., et al. 2004, ApJ, 607, 426

Koester, D., Weidemann, V., \& Zeidler, E. 1982, A\&A, 116, 147

Koester, D., Napiwotzki, R., Christlieb, N., et al. 2001, A\&A, 378, 556

Liebert, J., Harris, H. C., Dahn, C. C., et al. 2003, AJ, 126, 2521

Macdonald, J., Hernanz, M., \& Jose, J. 1998, MNRAS, 296, 523

McCook, G. P., \& Sion, E. M. 1999, ApJS, 121, 1

Pelletier, C., Fontaine, G., Wesemael, F., Michaud, G., \& Wegner, G. 1986, ApJ, 307,242

Press, W. H., Teukolsky, S. A., Vetterling, W. T., \& Flannery, B. P. 1992, Numerical recipes in FORTRAN, The art of scientific computing (Cambridge: University Press), 2nd edn.

Provencal, J. L., Shipman, H. L., Koester, D., Wesemael, F., \& Bergeron, P. 2002, ApJ, 568, 324

Scóccola, C., Althaus, L., Serenelli, A., Rohrmann, R., \& Corsico, A. 2006, A\&A, 451, 147

Thejll, P., Shipman, H. L., Macdonald, J., \& Macfarland, W. M. 1990, ApJ, 361, 197

Weidemann, V. 2005, in 14th European Workshop on White Dwarfs, ed.

D. Koester, \& S. Moehler, ASP Conf. Ser., 334, 15

Weidemann, V., \& Koester, D. 1995, A\&A, 297, 216 
D. Koester and S. Knist: New DQ white dwarfs in the SDSS, Online Material p 1

\section{Online Material}


D. Koester and S. Knist: New DQ white dwarfs in the SDSS, Online Material p 2

\section{Appendix A: Graphical display of spectral fits}

for $60 \mathrm{DQs}$

Thin blue line: observed spectra, thick red: model. Vertical axis is relative intensity on an arbitrary scale. The spectra are not in the same sequence as in Tables 1 and 2, since the intensity scales differ and similar spectra are grouped together. However, the objects can be cross-identified using the internal SDSS-MJDPLATE-FID designation on top of each panel. 
D. Koester and S. Knist: New DQ white dwarfs in the SDSS, Online Material p 3
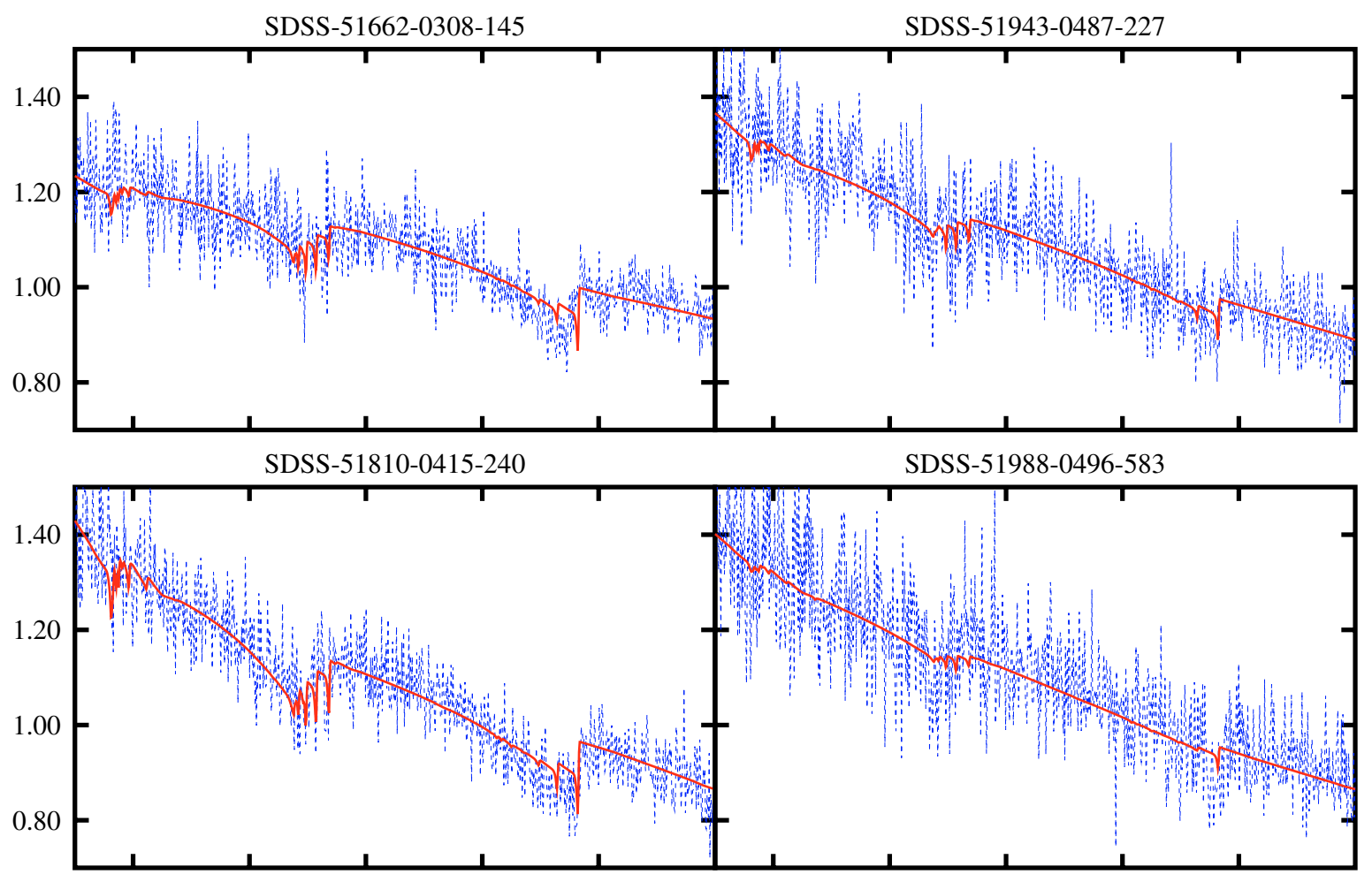

SDSS-51871-0403-268
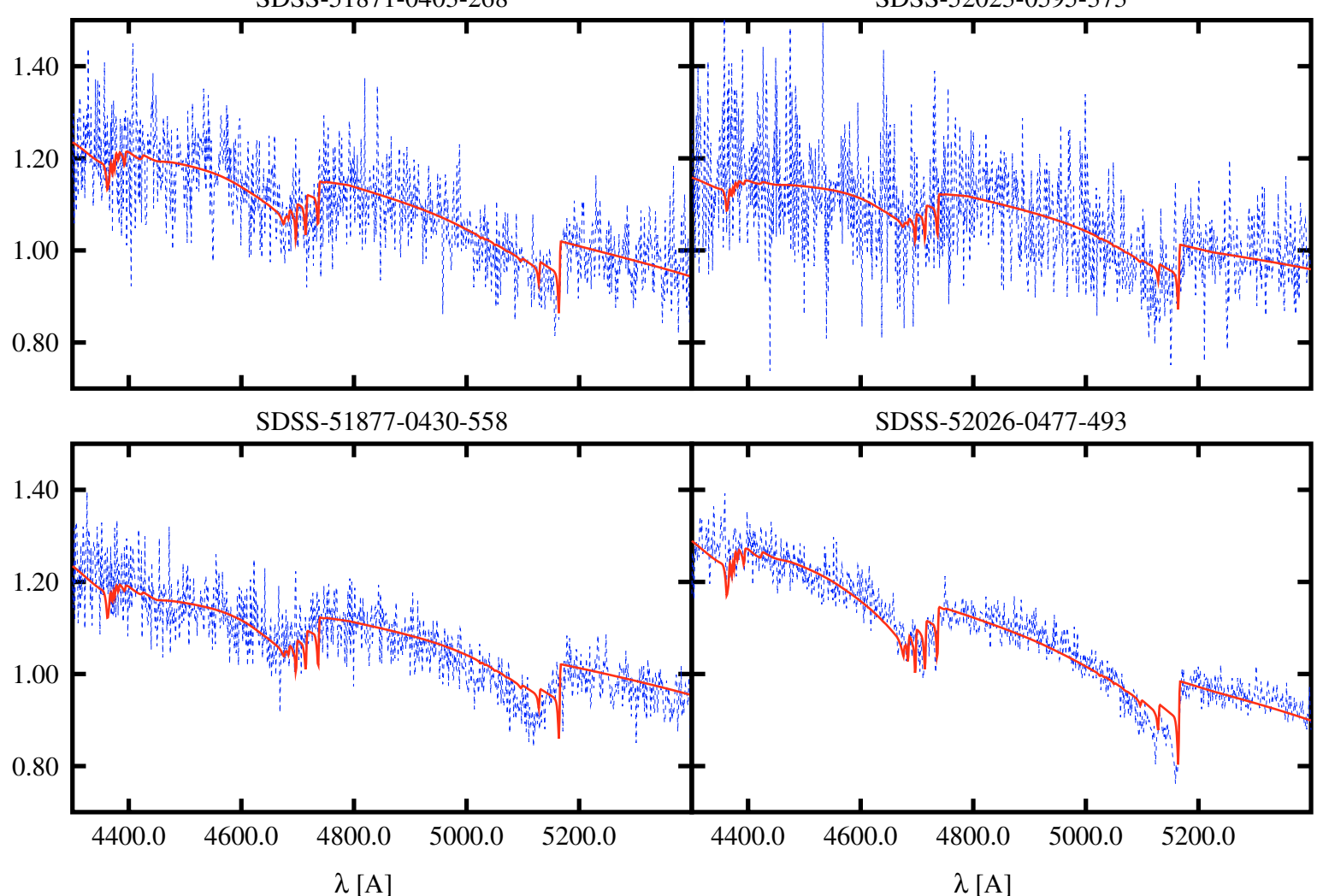

Fig. A.1. Spectral fit for DQ white dwarfs. 
D. Koester and S. Knist: New DQ white dwarfs in the SDSS, Online Material p 4
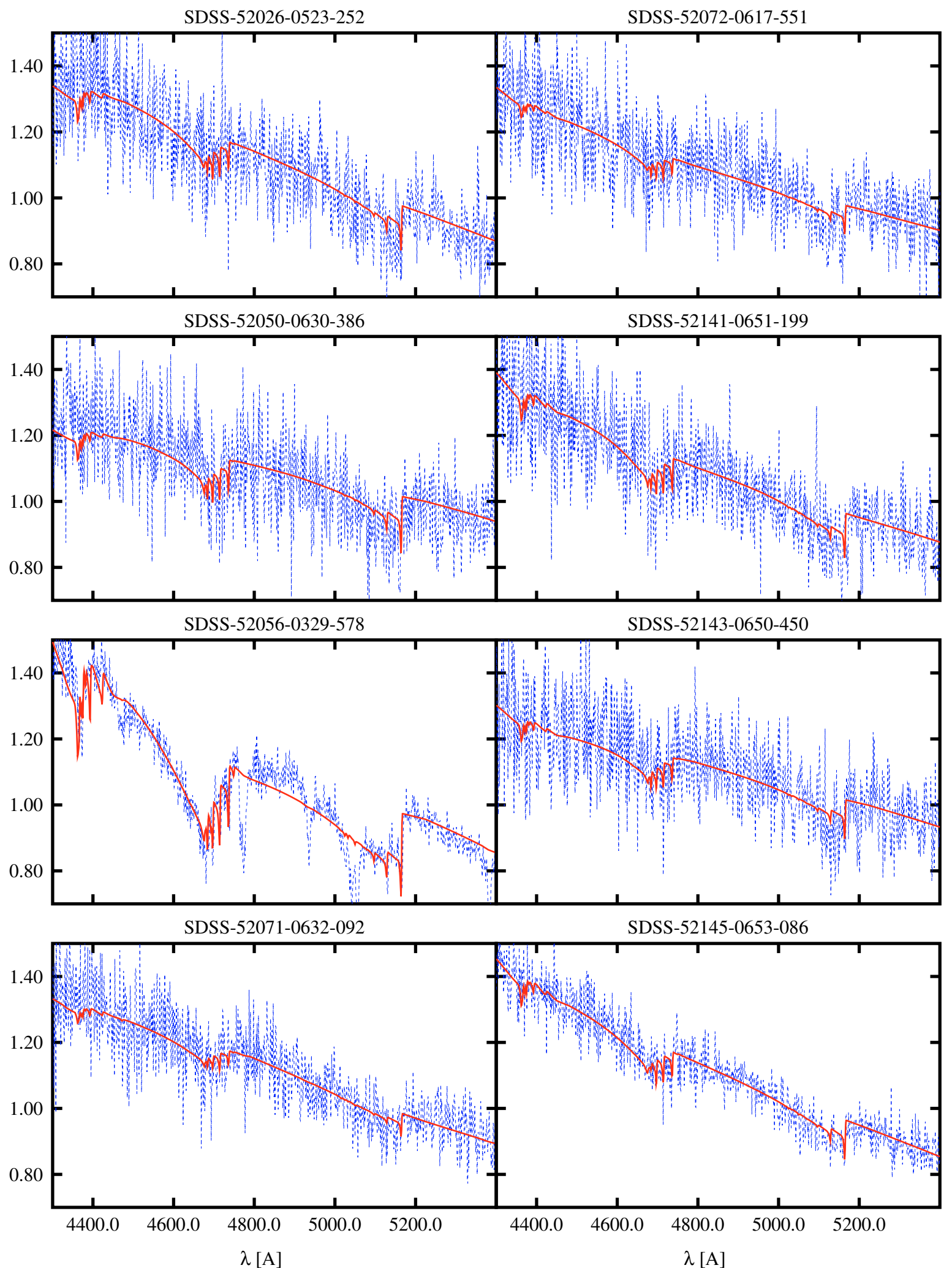

Fig. A.1. continued. 
D. Koester and S. Knist: New DQ white dwarfs in the SDSS, Online Material p 5
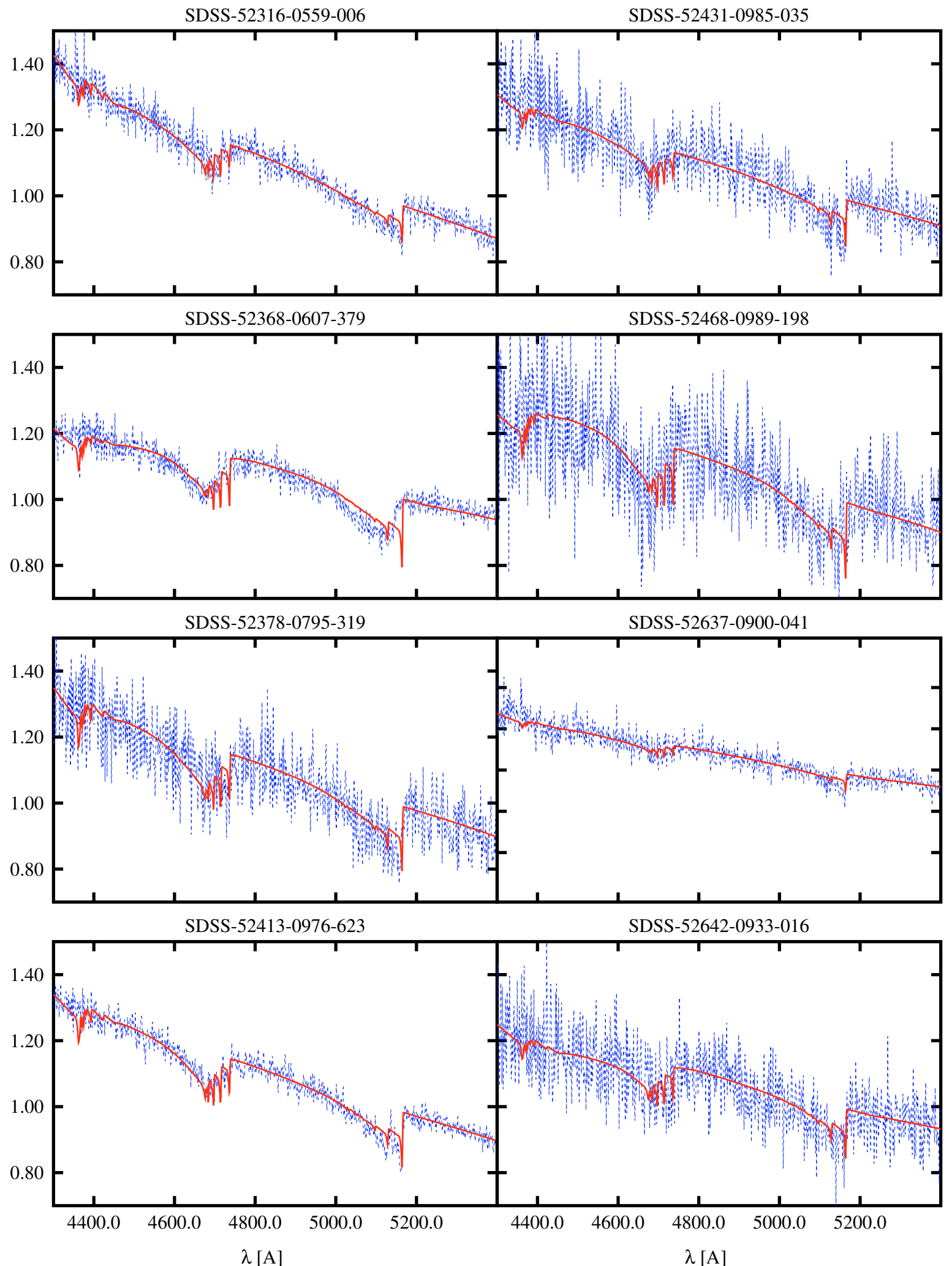

Fig. A.1. continued. 
D. Koester and S. Knist: New DQ white dwarfs in the SDSS, Online Material $p 6$
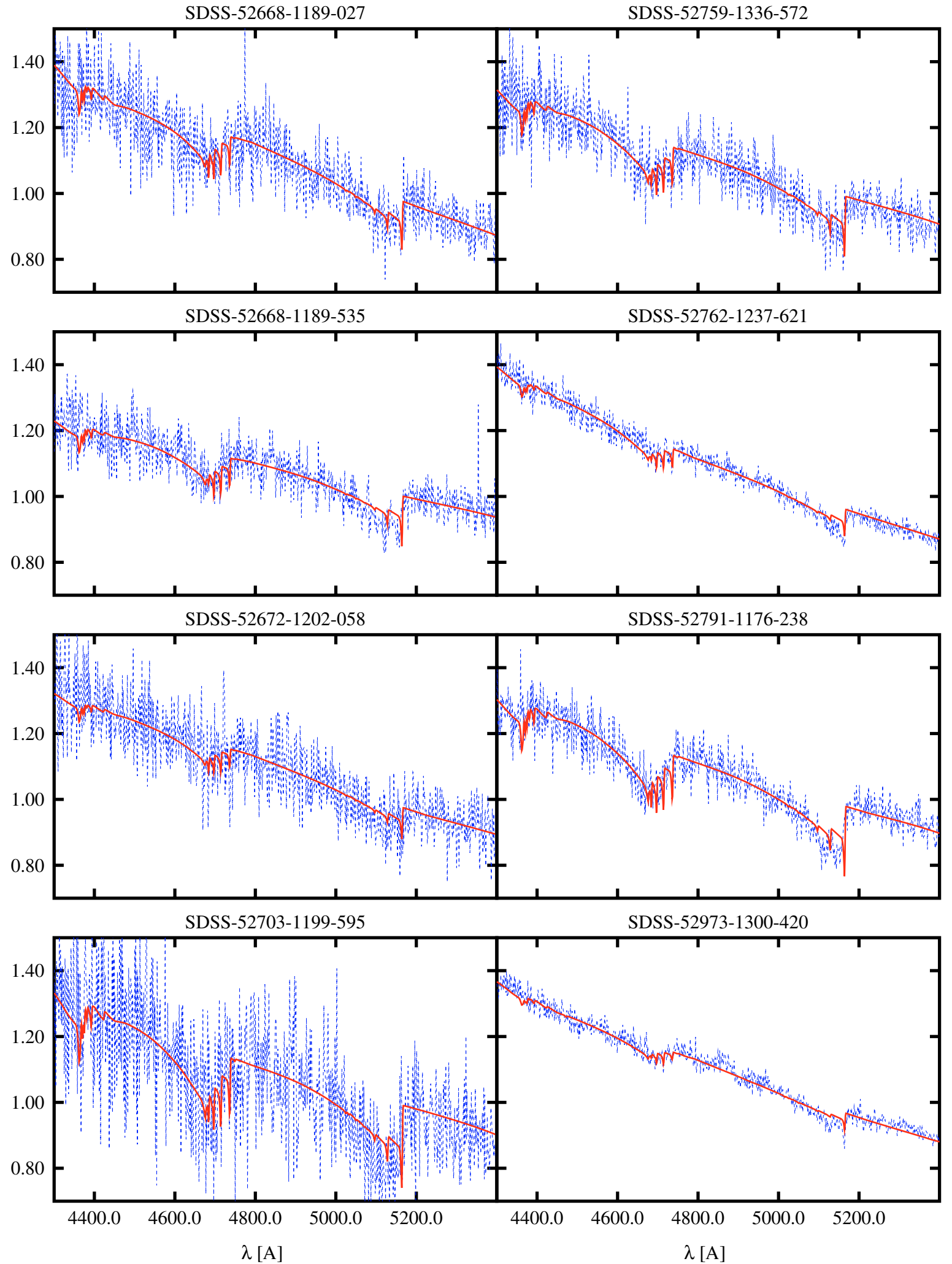

Fig. A.1. continued. 
D. Koester and S. Knist: New DQ white dwarfs in the SDSS, Online Material $p 7$
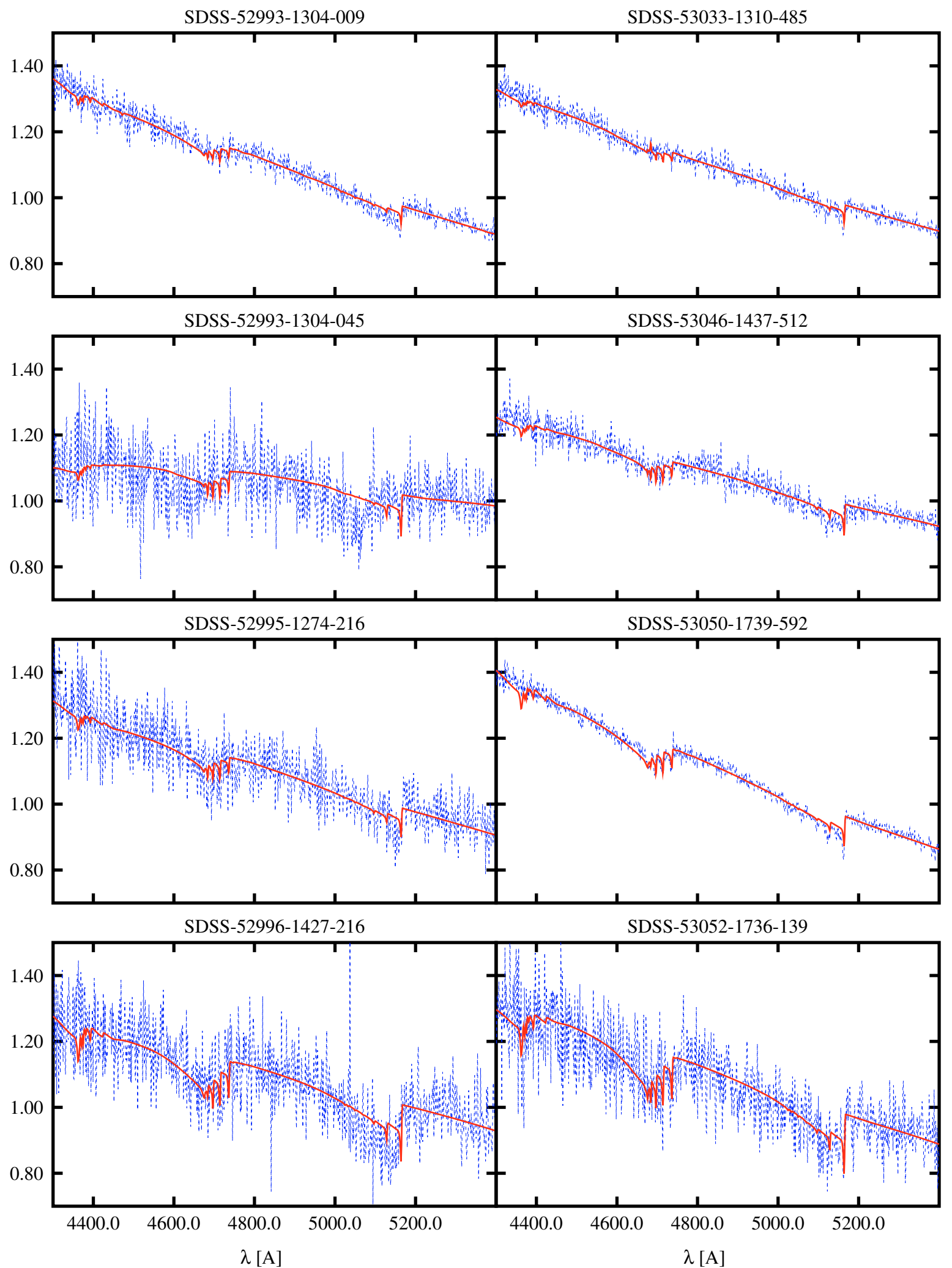

Fig. A.1. continued. 
D. Koester and S. Knist: New DQ white dwarfs in the SDSS, Online Material $p 8$
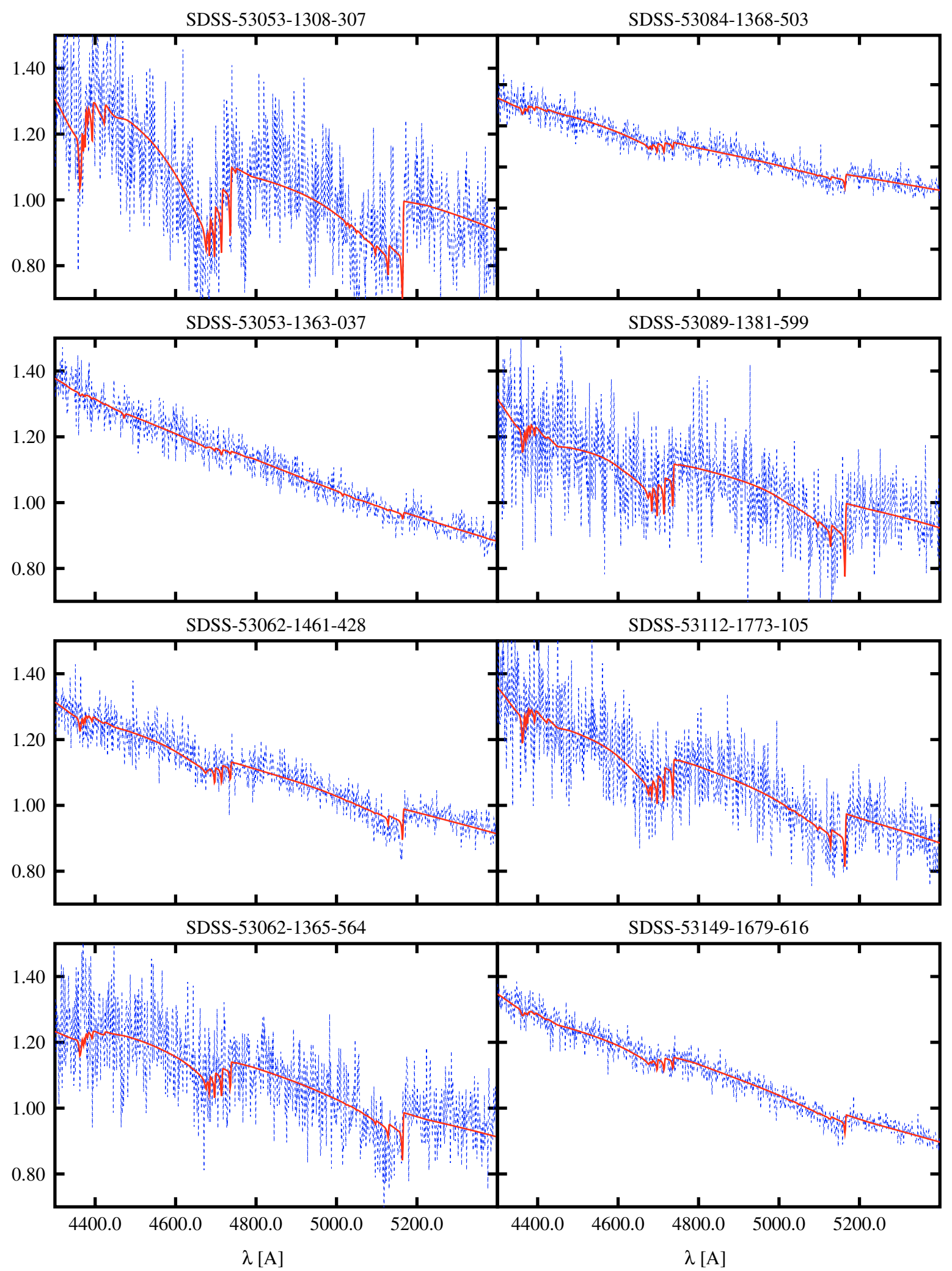

Fig. A.1. continued. 
D. Koester and S. Knist: New DQ white dwarfs in the SDSS, Online Material $p 9$

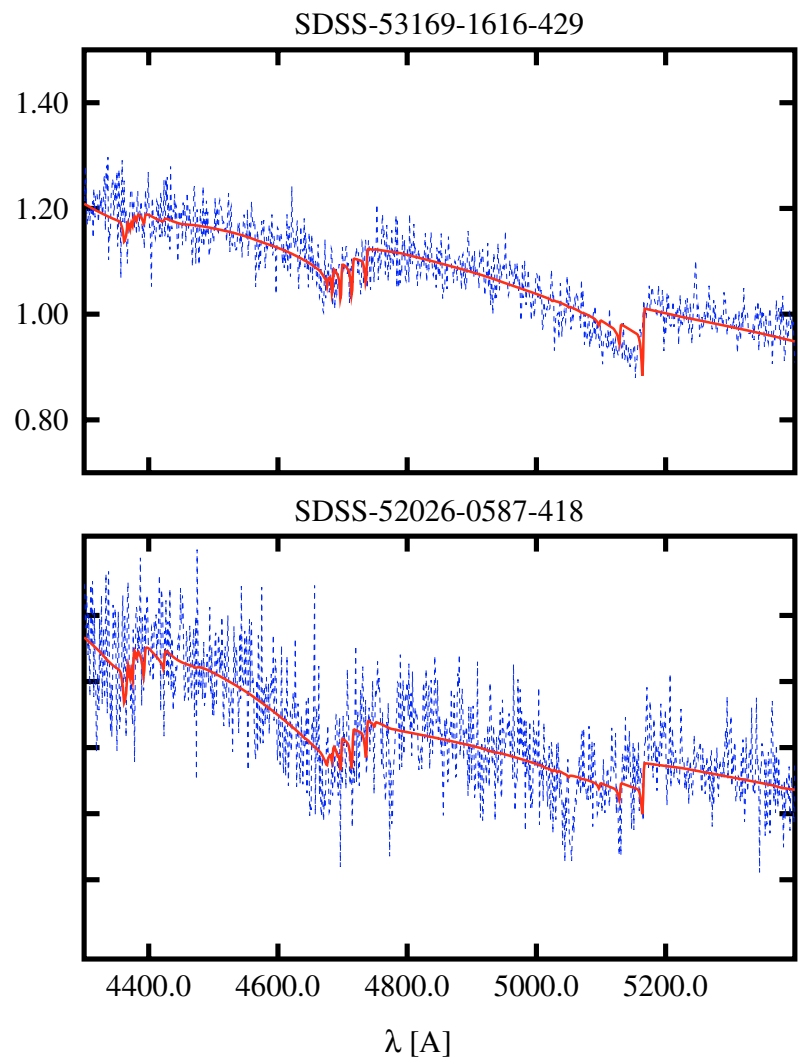

Fig. A.1. continued. 
D. Koester and S. Knist: New DQ white dwarfs in the SDSS, Online Material p 10
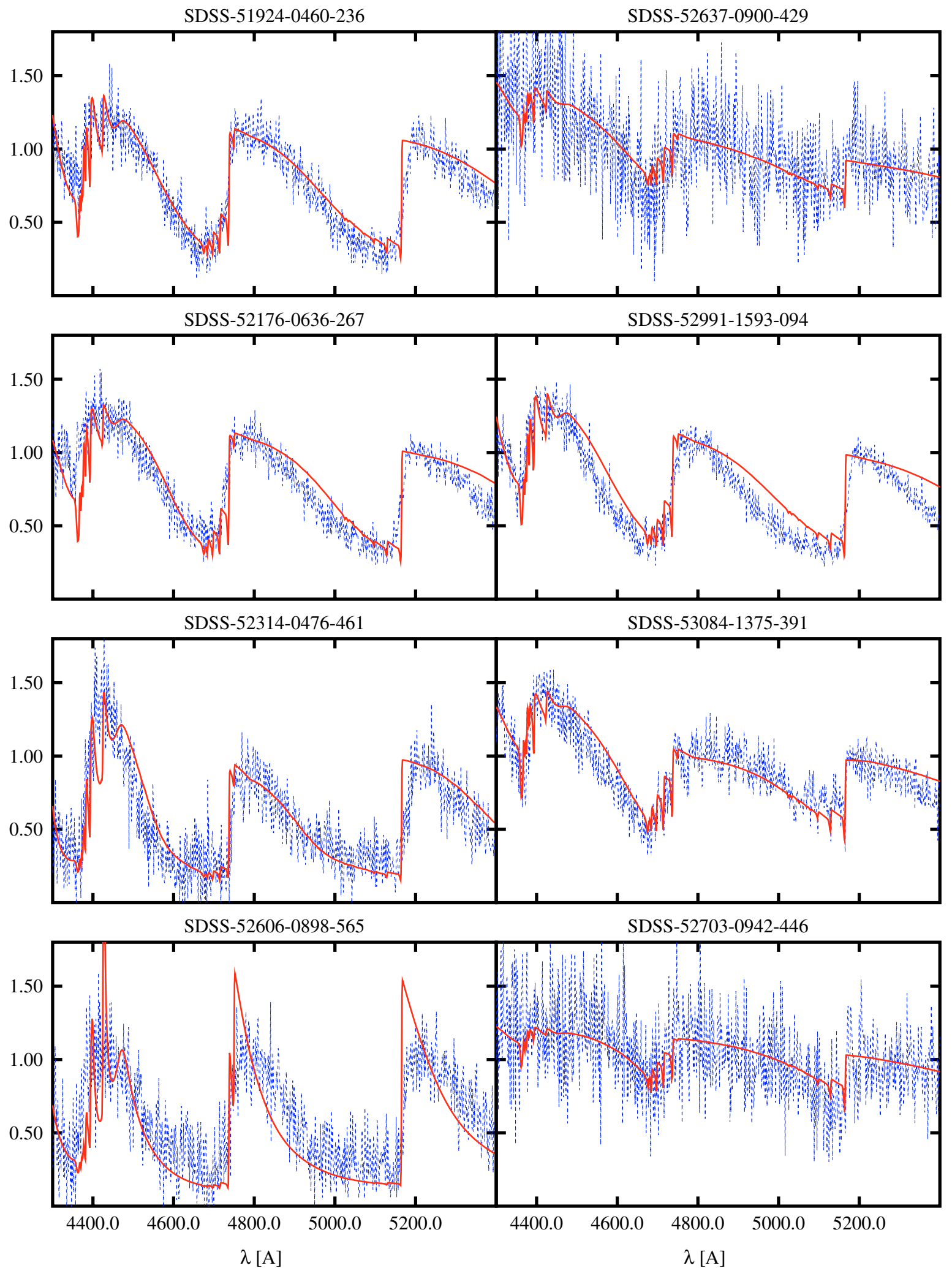

Fig. A.1. continued. 
D. Koester and S. Knist: New DQ white dwarfs in the SDSS, Online Material p 11

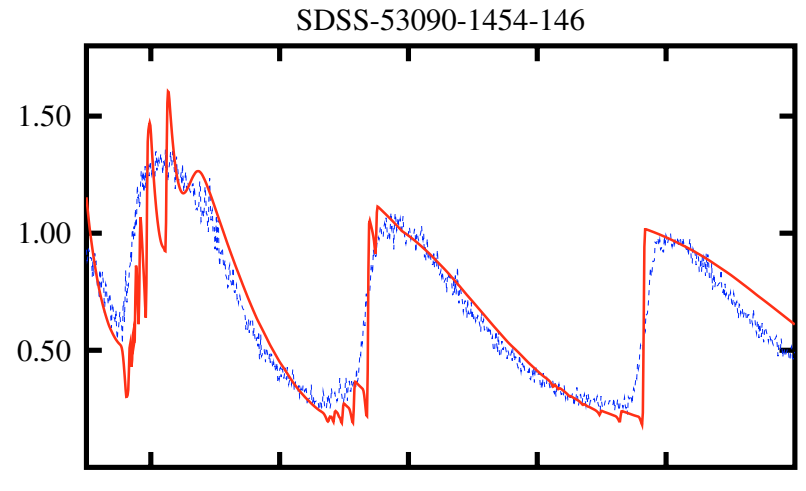

SDSS-53117-1451-035

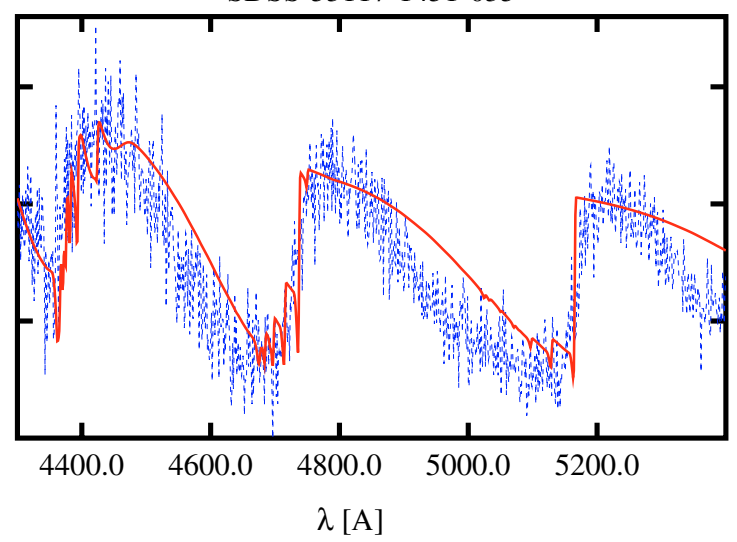

Fig. A.1. continued. 